\title{
In-Depth Analysis of Egg-Tempera Paint Layers by Multiphoton Excitation Fluorescence Microscopy
}

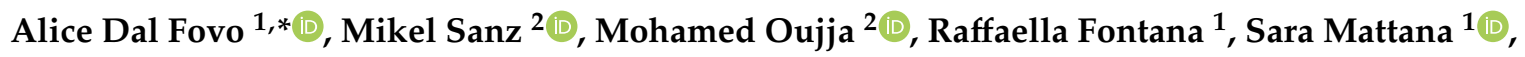 \\ Riccardo Cicchi ${ }^{1}{ }^{\circ}$, Piotr Targowski ${ }^{3}{ }^{\oplus}$, Marcin Sylwestrzak ${ }^{3}{ }^{\circledR}$, Aldo Romani ${ }^{4}$, Chiara Grazia ${ }^{4}$, \\ George Filippidis ${ }^{5}$, , Sotiris Psilodimitrakopoulos ${ }^{5}$, Andreas Lemonis ${ }^{5}$ and Marta Castillejo ${ }^{2}$ (1) \\ 1 Consiglio Nazionale delle Ricerche-Istituto Nazionale di Ottica, CNR-INO, Largo Enrico Fermi 6, \\ 50125 Firenze, Italy; raffaella.fontana@ino.cnr.it (R.F.); mattana@lens.unifi.it (S.M.); \\ riccardo.cicchi@ino.cnr.it (R.C.) \\ 2 Instituto de Química Física Rocasolano, CSIC, Serrano 119, 28006 Madrid, Spain; \\ mikel.sanz@iqfr.csic.es (M.S.); m.oujja@iqfr.csic.es (M.O.); marta.castillejo@iqfr.csic.es (M.C.) \\ 3 Institute of Physics, Faculty of Physics, Astronomy and Informatics, Nicolaus Copernicus University in \\ Torun, Grudziadzka 5, 87-100 Torun, Poland; ptarg@fizyka.umk.pl (P.T.); mars@fizyka.umk.pl (M.S.) \\ 4 Centro di Eccellenza SMAArt c/o Dipartimento di Chimica, Biologia e Biotecnologie, Via Elce di Sotto 8, \\ 06123 Perugia, Italy; aldo.romani@unipg.it (A.R.); chiara.grazia@unipg.it (C.G.) \\ 5 Institute of Electronic Structure and Laser (IESL), Foundation for Research and Technology (FORTH), \\ N. Plastira 100, 71110 Heraklion, Crete, Greece; filip@iesl.forth.gr (G.F.); sopsilo@iesl.forth.gr (S.P.); \\ leman@iesl.forth.gr (A.L.) \\ * Correspondence: alice.dalfovo@ino.it
}

Received: 27 March 2020; Accepted: 6 May 2020; Published: 8 May 2020

\begin{abstract}
The non-invasive depth-resolved imaging of pictorial layers in paintings by means of linear optical techniques represents a challenge in the field of Cultural Heritage $(\mathrm{CH})$. The presence of opaque and/or highly-scattering materials may obstruct the penetration of the radiation probe, thus impeding the visualization of the stratigraphy of paintings. Nonlinear Optical Microscopy (NLOM), which makes use of tightly-focused femtosecond pulsed lasers as illumination sources, is an emerging technique for the analysis of painted objects enabling micrometric three-dimensional (3D) resolution with good penetration capability in semi-transparent materials. In this work, we evaluated the potential of NLOM, specifically in the modality of Multi-Photon Excitation Fluorescence (MPEF), to probe the stratigraphy of egg-tempera mock-up paintings. A multi-analytical non-invasive approach, involving ultraviolet-visible-near infrared (UV-Vis-NIR) Fiber Optics Reflectance Spectroscopy, Vis-NIR photoluminescence, and Laser Induced Fluorescence, yielded key-information for the characterization of the constituting materials and for the interpretation of the nonlinear results. Furthermore, the use of three nonlinear optical systems allowed evaluation of the response of the analyzed paints to different excitation wavelengths and photon doses, which proved useful for the definition of the most suitable measurement conditions. The micrometric thickness of the paint layers, which was not measurable by means of Optical Coherence Tomography (OCT), was instead assessed by MPEF, thus demonstrating the effectiveness of this nonlinear modality in probing highly-scattering media, while ensuring the minimal photochemical disturbance to the examined materials.
\end{abstract}

Keywords: nonlinear optical microscopy; multi-photon excitation fluorescence; optical coherence tomography; egg-tempera paintings; non-invasive stratigraphy

\section{Introduction}

Nonlinear optical microscopy, in the modalities of Multi-Photon Excitation Fluorescence (MPEF) and Second and Third Harmonic Generation (SHG, THG), is based on nonlinear optical processes, in 
which multiple photons simultaneously interact with atoms or molecules of the irradiated material within the same quantum event. Such phenomena may be observed when a given material is excited by a tightly-focused near infrared femtosecond pulsed laser, propagating through a high numerical aperture microscope objective [1]. Nonlinear Optical Microscopy (NLOM) may provide compositional and structural information with micrometric axial resolution, based on the detection of fluorophores (by MPEF) [2], of crystalline or highly organized structures without inversion symmetry (by SHG) [3,4], and of local differences in refractive index and dispersion-i.e., interfaces between different materials (by THG) [5]. The application of NLOM was originally restricted to the biomedical field, mainly for in vivo imaging and mapping of subcellular structures such as stacked membranes, arranged proteins (e.g., collagen) [6], and dipolar molecules [7]. Specifically, since 1990 [8], MPEF has been playing a pivotal role in the study of biological matter, in conjunction with confocal microscopy, for a variety of applications ranging from the tracking of individual molecules within living cells to the observation of whole organisms [9].

In more recent years, the promising results provided by NLOM have encouraged its implementation in the diagnosis of Cultural Heritage $(\mathrm{CH})$ for the non-destructive analysis of several types of samples and artworks $[10,11]$. The increasing use of NLOM is due to the several advantages offered by this technique, which may allow it to overcome the detection limits of other more established ones, such as Optical Coherence Tomography (OCT) [12,13]. Specifically, OCT has proven particularly effective in the cross-sectional analysis of varnish layers, even highly reflective ones [14], in some cases also enabling the discrimination between aged and new varnishes [15]. However, the two major observed drawbacks of this technique are the limited probing depth in highly turbid media, such as paint layers [16], and the need to apply complementary techniques to chemically characterize the examined material [17]. By contrast, NLOM may provide structural, morphological, and compositional information in one single measurement, based on the simultaneous acquisition of different nonlinear signals (MPEF, SHG, THG) generated inside the material through interaction with the tightly focused femtosecond laser. Furthermore, the nonlinear dependence of the processes on the intensity of the excitation laser entails intrinsic sub-diffraction limited axial resolution, as all nonlinear interactions are confined within the focal volume of the laser beam (with dimensions in the micron range). In such a manner, photo-bleaching phenomena and out-of-focus damages are drastically diminished, which is a priority for $\mathrm{CH}$ studies. Among nonlinear processes, the minimal disturbance to the analyzed specimen is ensured by nonlinear scattering (SHG and THG), in which no energy is deposited in the medium [18]. As regards nonlinear absorption processes (MPEF), safe measurement conditions can be achieved by keeping the laser power within specific limits that are related to the optical properties and chemical composition of each material. More specifically, by monitoring the photo-damage induced by MPEF upon repetitive irradiation on a series of modern acrylic painted samples, it was highlighted that the photon dose used for irradiation is a crucial parameter for preventing damage [19]. The power threshold-i.e., the laser power at which the fluorescence intensity starts to decrease after consecutive irradiations-sets the limit for safe cross-sectional imaging of paint layers by MPEF, while still gathering enough nonlinear optical signals at a suitable signal-to-noise ratio. Moreover, it has to be underlined that, thanks to the use of near infrared laser wavelengths, the probing capability of NLOM is generally high in most pictorial materials. Besides, the possibility to perform MPEF measurement in the reflection mode enlarges its applicability to a wide range of real cases, i.e., painting materials lying on opaque substrates (board, wood, canvas, etc.). In order to enable in situ analysis on artworks, the development of a transportable prototype system is also envisaged. The design and construction project of a transportable nonlinear microscope are described in detail in [20].

The potential of NLOM in CH applications was initially tested for the three-dimensional (3D) imaging of protective layers, in support of the cleaning operation performed on natural and synthetic varnishes, which irreversibly modifies the morphology and thickness of the superficial layers [21,22]. It was demonstrated that NLOM makes the in-depth monitoring of varnish degradation due to aging [23] or laser ablation [24] feasible. Furthermore, wooden artefacts were analyzed through SHG 
and MPEF, enabling both the imaging and the chemical characterization of wood microstructures [3]. Silver-based objects were also studied by means of MPEF imaging in order to identify and quantify the presence of corrosion layers [25]. In the last few years, pictorial layers were examined with the nonlinear modality of femtosecond pump-probe microscopy [26]. MPEF was also tested on paint layers [2] for the monitoring of the laser-removal of organic and inorganic acrylic paints [21] and for the evaluation of paint layer thickness with micrometric resolution $[27,28]$. As pointed out by these previous studies, the presence of highly scattering and/or absorbing media (pigments) in multi-layered painted systems tends to obstruct the detection of the NLOM signal, thus impeding the visualization of the stratigraphy. Specifically, MPEF imaging, performed with a laser wavelength set at $740 \mathrm{~nm}$ on the same set of samples analyzed in this study [28], enabled the visualization of the micromorphology of the painting surfaces but provided underestimated thickness values of the paint layers with respect to the real ones. Based on these results, it was hypothesized that a longer excitation wavelength could provide more reliable cross-sectional measurements. Recently, it has been demonstrated that NLOM with excitation of $800 \mathrm{~nm}$ in the MPEF modality serves to determine the thickness of single-layered samples (acrylic paints laid on glass support) [27], despite the severe drop in signal intensity with increasing depth. This suggests that the in-depth measurement of paint layers is feasible, provided that paint materials have a sufficient degree of transparency to the excitation wavelength and in the spectral range of the emitted fluorescence.

In this work, we assessed the capabilities of the NLOM modality of MPEF for the micrometric surface mapping and the in-depth profiling of thin egg-tempera layers, using suitable measurement conditions to ensure safe analysis of the sensitive paints. The samples, designed to simulate real egg-tempera paintings on wooden support, were non-invasively characterized through the combined application of well-established linear optical techniques, including ultraviolet-visible-near infrared (UV-Vis-NIR) Fiber Optics Reflectance Spectroscopy (FORS) [29], Vis-NIR photoluminescence (PL) [30], and Laser Induced Fluorescence (LIF) [31]. The optical characterization was used to address and interpret the nonlinear results obtained by MPEF, and the comparison of MPEF with OCT measurements allowed the potential of NLOM in the 3D survey of highly scattering painting media to be highlighted.

\section{Samples}

The samples examined in this study are shown in Figure 1 (a detailed description of the samples can be found in [28]). Three pigments by @Kremer (Germany) were selected for the paint layers due to their wide use in historical artworks, and also because of their specific optical properties. These were red lead $\left(\mathrm{Pb}_{3} \mathrm{O}_{4}\right.$, cod. 42500), cadmium yellow medium (cadmium sulphide, $\mathrm{CdS}$, cod. 21051), and Egyptian blue (blue copper silicate, chemically classified as the mineral cuprorivaite, $\mathrm{CaCuSi}_{4} \mathrm{O}_{10}$, cod. 10060). Pigments in the form of powder were finely ground and then mixed with the binder; beaten and filtered egg yolk. The analyzed samples, labelled R, Y, and B (red, yellow, and blue, respectively), consisted of a sequence of material layers as follows (from bottom to top): wooden support (a "sandwich" structure made of fir and poplar), preparation layer (sifted gypsum dispersed in a solution of rabbit skin glue and water), underdrawings (lead and tin stylus, natural carbon, iron-gall ink), and pictorial layer (egg-tempera with the three different pigments).
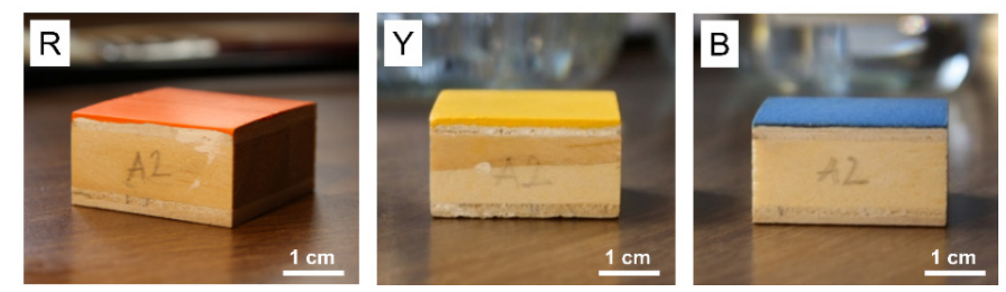

Figure 1. Red, yellow, and blue (, ,, and $B$ ) samples simulating egg-tempera painting on wooden support. 


\section{Methods}

\subsection{UV-Vis-NIR Fibre Optics Reflectance Spectroscopy}

Fiber Optics Reflectance Spectroscopy (FORS) was performed with a Zeiss Multi-Channel Spectrometer, available at the Istituto Nazionale di Ottica (INO), which included a MCS 521 VIS NIR-extended module and a MCS 511 NIR 1.7 module with spectral sensitivity in the 304-1100 nm and $950-1700 \mathrm{~nm}$ ranges (spectral resolution of $3.2 \mathrm{~nm}$ and $6.0 \mathrm{~nm}$ in the visible and NIR region, respectively). The illumination/observation geometry was $45^{\circ} / 0^{\circ}$. Each FORS spectrum was the average of nine measurements performed in three different positions on each sample. The output signal was processed with dedicated software.

\subsection{Vis-NIR Photoluminescence}

Photoluminescence (PL) measurements were performed at the University of Perugia (UNI-PG) using two high-sensitivity calibrated charge-coupled device (CCD) spectrometers. The first was an Avaspec-ULS2048 XL-RS-USB2 (300-1150 nm sensitivity range, spectral resolution $9.2 \mathrm{~nm}$ with $200 \mu \mathrm{m}$ slit) and the second was an AvaSpec-NIR256-1.7TEC, equipped with two ultra-compact laser diodes (Toptica Photonics AG, D, operating at 375 and $445 \mathrm{~nm}$ ) and a pulsed Nd:YAG laser (Alphalas, D, providing the 4 th harmonic frequency-i.e., $266 \mathrm{~nm}$ ). The three different excitation wavelengths were selected with the aim of approximately reproducing, in a one-photon absorption scheme, the excitation conditions of nonlinear absorption (of 2 or 3 photons) of the fs-laser wavelengths used for MPEF (800, $1028 \mathrm{~nm}$ ). The output power was measured with an irradiance-calibrated AvaSpec-2048-2 spectrometer (Avantes, NL; 171-1100 $\mathrm{nm}$ range, spectral resolution, $1.2 \mathrm{~nm}$ ) equipped with a $200 \mu \mathrm{m}$ diameter optical fiber and an $8 \mathrm{~mm}$ active area cosine corrector placed at the sample position. The powers used were $109 \mu \mathrm{W}$ for the laser diodes (375 and $445 \mathrm{~nm}$ ) and $40 \mu \mathrm{W}$ for the pulsed Nd:YAG laser (266 nm). The spot size at the sample position was approximately $1.8 \mathrm{~mm}^{2}$. The PL profiles collected by the AvaSpec-NIR256-1.7TEC spectrometer were scaled to match the Avaspec-ULS2048 XL-RS-USB2 data in the $950-1000 \mathrm{~nm}$ range.

\subsection{Laser Induced Fluorescence}

Laser Induced Fluorescence (LIF) spectra were collected at the Instituto de Química Física Rocasolano (IQFR) using laser excitation wavelengths of 355 and $266 \mathrm{~nm}$ (3rd and 4th harmonic of a Q-switched Nd:YAG laser, $15 \mathrm{~ns}$ pulses, $1 \mathrm{~Hz}$ repetition rate) and a $0.30 \mathrm{~m}$ spectrograph with a 300 lines/mm grating (TMc300 Bentham) coupled to an intensified charged coupled detector (ICCD) (2151 Andor Technologies). The acquisition of the LIF signal was temporally gated with zero-time delay with respect to the arrival of the pulse to the surface of the sample and a width of $1 \mu \mathrm{s}$. The sample was illuminated with a $1.6 \mathrm{~mm}^{2}$ (ellipsoidal shape) laser spot at an incidence angle of $45^{\circ}$ with pulses of around $1 \mathrm{~mJ}$. For the results presented here, a $300 \mathrm{~nm}$ cut-off filter was set in front of the spectrograph to block the second order signal. Each spectrum resulted from the accumulation of 25 measurements.

\subsection{Optical Coherence Tomography}

The analysis was performed at Nicolaus Copernicus University with a Spectral Domain (SD) OCT prototype [32]. This high-resolution portable device comprises a broad-band light source made up of super luminescent LEDs emitting in the 770-970 $\mathrm{nm}$ band. The radiation intensity at the surface of the object never exceeded $1 \mathrm{~mW}$, and it was focused at any given spot on the object for only $40 \mathrm{~ms}$ due to the fast scanning time. To obtain the exploitable signal, light scattered and/or reflected by the elements in the object's structure interferes with light coming from the reference arm and transferred (as always for SDOCT) to a spectrograph. In these experiments, a laboratory built device (with volume phase holographic transmission grating optimized for $850 \mathrm{~nm}, 1200$ grooves/mm from Wasatch Photonics, and a single line CCD camera AVIIVA EM4 from e2v) was used. 
The lateral imaging resolution is about $12 \mu \mathrm{m}$, whereas the axial one is $3.3 \mu \mathrm{m}$ in air, with an axial imaging range of $1.4 \mathrm{~mm}$. Depths values in OCT tomograms represent optical distances, therefore real axial distances within the imaged structure may be obtained by dividing the apparent thickness by the refractive index of the medium. Therefore, the axial resolution in a transparent material (e.g., varnish layer with a refractive index of 1.5) may be estimated at around $2.2 \mu \mathrm{m}$. The distance between the object and the device was $43 \mathrm{~mm}$, and during a single measurement an area of $5 \times 10 \mathrm{~mm}^{2}$ was scanned in about $12 \mathrm{~s}$ for a total of 50 axial scans for each sample. The images in Section 4.4 (Figure 5) have been vertically stretched twelve-fold for better readability.

\subsection{Nonlinear Optical Microscopy via Multi-Photon Excitation Fluorescence}

Three different nonlinear optical microscopes were used for MPEF analysis. In all three systems the laser spot had a diameter of around $1 \mu \mathrm{m}$ in the focal plane. MPEF imaging was performed using the device developed at the Institute of Electronic Structure and Laser-Foundation for Research and Technology (IESL-FORTH), consisting of a modified inverted Zeiss Axio Observer Z1 microscope and a pair of galvanometric scanning mirrors from Cambridge Technology for raster-scanning. A description of the apparatus can be found in [33,34]. The excitation source was a Yb:KGW Pharos SP laser from Light Conversion, centered at $1028 \mathrm{~nm}$ and delivering pulses of $90 \mathrm{fs}$ at a repetition rate of $76 \mathrm{MHz}$. The objective lens used for the excitation and collection of the nonlinear signals was a Zeiss Plan-Apochromat $20 \times 0.8$ NA. In this system, damage of the samples upon NIR excitation was avoided by employing average powers below $25 \mathrm{~mW}$ at the sample plane [2]. All images were acquired in the backward direction, collecting the signals in the visible region of the spectrum. The emitted fluorescence was recorded using a photomultiplier tube (PMT) (Hamamatsu H9305-04). A short-pass filter (FF01-790/SP, Semrock) was placed in front of the PMT to cut off the reflected laser light and solely detect the fluorescence arising from the samples. Typical time duration for obtaining a two-dimensional $500 \times 500$ pixel MPEF image was approximately one second. The photon dose, namely the number of laser pulses per irradiated point on the sample surface, was 320 pulses/point.

The second device used was a nonlinear optical microscope developed at IQFR, which allows for the point-wise collection of MPEF signals in epi-detection mode. A detailed description of this device can be found in $[19,27]$. Briefly, the excitation light source is a mode-locked Ti:Sapphire femtosecond laser emitting at $800 \mathrm{~nm}$, releasing $70 \mathrm{fs}$ pulses at a repetition rate of $80 \mathrm{MHz}$. The laser beam passes through a set of two continuously variable metallic neutral density filters arranged in a serial mode (Thorlabs, NDC-50C-2M) in order to get average powers below $5 \mathrm{~mW}$ (which is below the damage threshold of the paints [19]). The laser beam was focused on the sample by a microscope objective lens (M Plan Apo HL 50X, Mitutoyo, NA 0.42). The MPEF signals were collected in the backward direction through the microscope objective and a beam splitter (70/30) and measured using a photomultiplier tube (9783B, ET Enterprises) connected to a lock-in amplifier (SR810 DSP, Stanford Research Systems) to ensure high amplification and signal-to-noise ratio. A short pass filter (335-610 nm, Thorlabs FGB37S) was placed at the entrance of the photomultiplier to cut off the reflected laser light. The remaining $30 \%$ of the MPEF signal was sent to a CCD camera (Thorlabs DCC1645C) for online visualization of the sample surface. The photon dose applied on the sample surface was $80 \times 10^{6}$ pulses/point.

The third system, a custom-made laser-scanning nonlinear optical microscope developed at INO, is described in detail elsewhere $[19,35,36]$. The source is an optical parametric oscillator OPO pumped by a doubled $\mathrm{Yb}$-based pulsed laser with $80 \mathrm{MHz}$ repetition rate (Chameleon Discovery, Coherent). For this application, the excitation wavelength was fixed at $800 \mathrm{~nm}$ with short pulses (100 fs). The beam was focused on the sample by an air-immersion Plan-Apochromat $10 \times$ objective lens, (NA, 0.45; WD, $2.1 \mathrm{~mm}$, Carl Zeiss Microscopy) mounted on a piezoelectric scanning system for axial positioning (P-725KHDS PIFOC, Physik Instrumente). The MPEF signal was acquired in epi-detection and measured using a photomultiplier tube (H7422-40, Hamamatsu) with sensitivity in the spectral range of $360-660 \mathrm{~nm}$, upon proper filtering. The applied photon dose was 200 pulses/point, and the laser power was set below $10 \mathrm{~mW}$ to ensure safe measurement conditions. 


\section{Results}

\subsection{UV-Vis-NIR Fiber Optics Reflectance Spectroscopy}

FORS spectra are shown in Figure 2 and were used to characterize the optical properties of the three paint systems in view of the following MPEF analysis. Red lead shows a typical sigmoidal shape with an inflection point at $570 \mathrm{~nm}$, which corresponds to an indirect band gap of about $2.2 \mathrm{eV}$ [37]. A sigmoidal shape is also observed in the reflectance profile of the semiconductor cadmium yellow [38,39], with an inflection point at $530 \mathrm{~nm}$. Reflectance profiles collected on the Egyptian blue sample show absorption bands in the Vis-NIR range. Specifically, the shoulder at $540 \mathrm{~nm}$ and the two bands centered at about 630 and $795 \mathrm{~nm}$ are attributable to three electronic transitions $\left(2 \mathrm{~B}_{1 \mathrm{~g}} \rightarrow 2 \mathrm{~B}_{2 \mathrm{~g}}, 2 \mathrm{E}_{\mathrm{g}}, 2 \mathrm{~A}_{1 \mathrm{~g}}\right)$ related to $\mathrm{Cu}^{2+}$ ions in an octahedral ligand field with a square-planar $\left(\mathrm{D}_{4 \mathrm{~h}}\right)$ symmetry $[40,41]$.

Within the spectral range of interest, in which the absorption of the IR excitation wavelength and the emission of the MPEF signal are expected, red lead and cadmium yellow show similar behavior. In fact, both paints exhibit an absorption band between 350 and $550 \mathrm{~nm}$, with a following rapid increase of the reflectance (probably due to the gypsum background), reaching 90\% at around 600-650 nm and remaining between $100 \%$ and $80 \%$ up to $1300 \mathrm{~nm}$. With reference to the Egyptian blue, the reflectance spectrum shows a low reflectance at $800 \mathrm{~nm}$ (about 15\%), which increases at $1028 \mathrm{~nm}(\mathrm{R}=30 \%)$. These variable absorption and reflectance features represent favorable conditions for both the excitation and signal collection through MPEF.

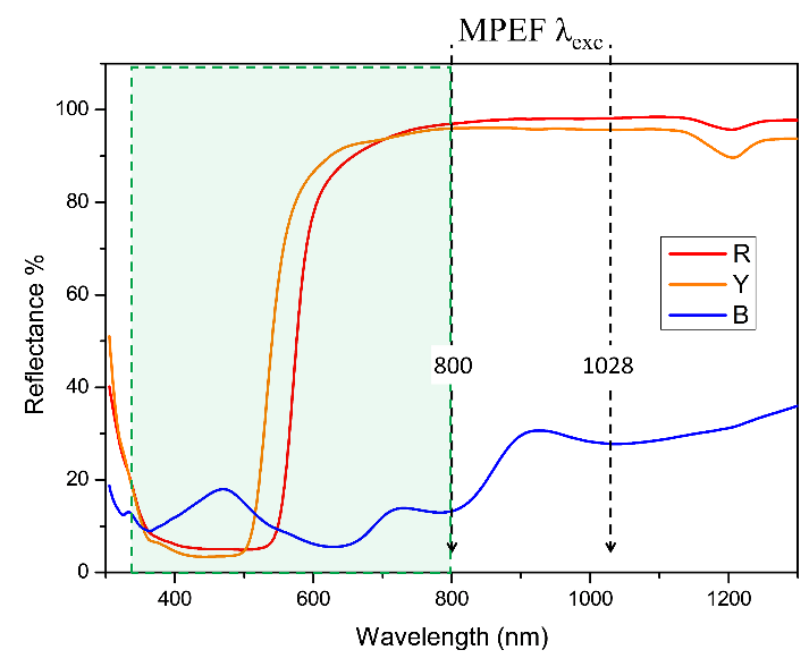

Figure 2. Reflectance spectra of red (R), yellow (Y), and blue (B) tempera paint samples. The excitation wavelengths (800 and $1028 \mathrm{~nm}$ ) and the spectral range for detection used for Multi-Photon Excitation Fluorescence (MPEF) measurements (335-800 nm, dashed green rectangle) are highlighted in the reflectance spectra.

\subsection{Vis-NIR photoluminescence}

The spectral identification of the PL emission from the three tempera samples $(R, Y$, and $B)$ is particularly relevant to ensure the proper detection range of the MPEF signals attributed to the paint layer. The PL was measured upon excitation at 266, 375, and 445 wavelengths, which were chosen to characterize the fluorescence spectra that would be expected under two- and three-photon absorption at the two NIR wavelengths of the femtosecond laser sources used here (800 and $1028 \mathrm{~nm}$ ). The detection range of PL analysis, 300-1600 nm, includes the spectral windows for the detection of the MPEF signals (see Section 3.5). The results of these measurements are reported in Figure 3. Inorganic pigments are generally characterized by a low fluorescence quantum yield, as can be observed in the data reported. Red lead, specifically, is an indirect semiconductor, and thus it is not considered an efficient fluorescent emitter. The very weak PL signals recorded on the red sample (Figure 3) are ascribable to a weak 
luminescence of the pigment at around $600 \mathrm{~nm}$, which could be assigned to the Near Band Edge (NBE) emission of the semiconductor, although previous studies [42] associate emission in this region with irregularities in the crystal structure or the presence of impurities. The PL of this sample also includes the contribution of the binder emission, centered at about $450 \mathrm{~nm}$, whose longer wavelength could be partially filtered by pigment absorption [43-47].

Cadmium pigments are generally characterized by radiative recombination with the NBE emission of fluorescence in the visible range, as well as emission attributed to Deep Level Emissions (DLE) in the near infrared range. The weak PL band in the visible range observed for the yellow tempera paint is red-shifted with respect to the absorption edge, which is likely due to the cadmium yellow pigment, which filters the emission of the organic binder and/or the contribution of the excitation source [48]. By using the 375 and $445 \mathrm{~nm}$ excitation wavelengths, the DLE emission of the cadmium-based pigments is clearly detectable and characterized by a shoulder at $760 \mathrm{~nm}$ and a maximum at $1020 \mathrm{~nm}$. This emission is generally more detectable at the low excitation fluences used for PL measurements, while, at higher fluences, the saturation of the defects from which the DLE is originated induces an enhancement of the NBE signal, as shown in the LIF measurements (see below).

Photoluminescence profiles of Egyptian blue show an intense emission band centered at about $910 \mathrm{~nm}$ attributable to the lowest energy electronic transition $\left(2 \mathrm{~B}_{2 \mathrm{~g}}-2 \mathrm{~B}_{1 \mathrm{~g}}\right)$ [41]. Upon excitation at $266 \mathrm{~nm}$, a severe decrease of the emission intensity is observed in the blue sample, mainly due to the competitive absorption of a relevant portion of the excitation light by the binder.

The reflectance spectra measured in the spectral detection range of the nonlinear optical microscopes used in this study (reported in Section 4.1) clearly indicate that the three paint systems display absorption bands in the region of their fluorescence emission range determined by PL. Hence, the possible reduction of the MPEF signal by auto-absorption has to be taken into account during NLOM analysis.

a)

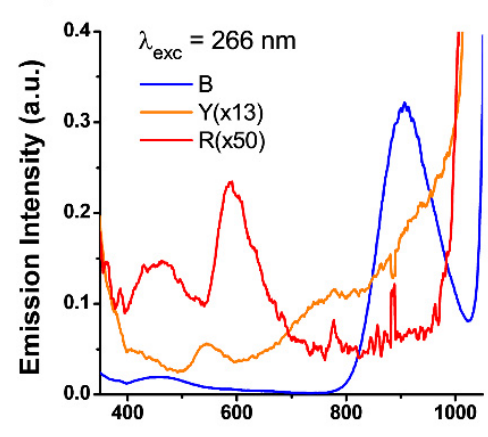

b)

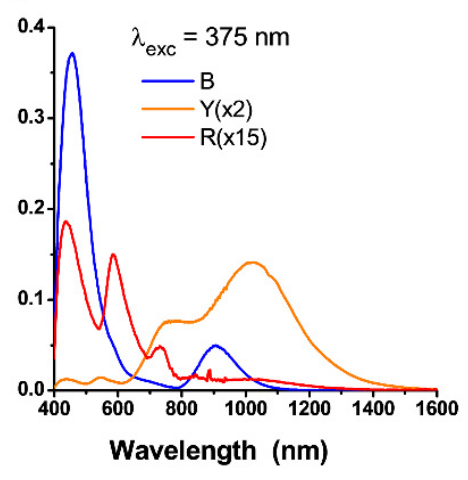

c)

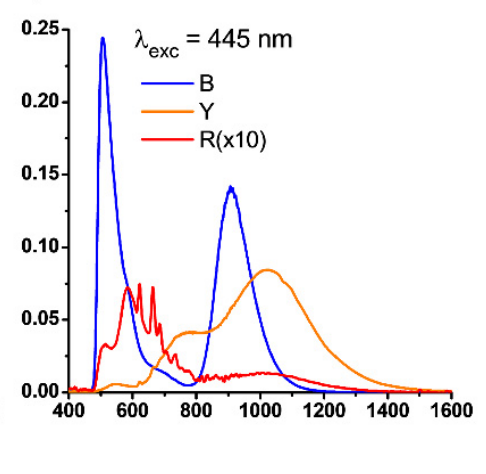

Figure 3. Fluorescence spectra of red, yellow, and blue samples, acquired after excitation at $266 \mathrm{~nm}(\mathbf{a})$, $375 \mathrm{~nm}(\mathbf{b})$, and $445 \mathrm{~nm}$ (c).

\subsection{Laser Induced Fluorescence}

The fluorescence behavior of the three pigments was further examined with LIF using two excitation wavelengths, i.e., 266 and $355 \mathrm{~nm}$. Signals were acquired in the 300-700 nm spectral range, in consistency with the detection range of the nonlinear optical microscopes. As for PL analysis, the two excitation wavelengths used for LIF are expected to produce similar fluorescence than those collected by three-photon absorption at 800 and $1028 \mathrm{~nm}$, respectively.

To facilitate analysis of the results, LIF spectra of all samples acquired at the same excitation wavelengths were normalized to the maximum intensity and displayed together in two graphs (Figure 4a,b). The common broad emission band between 400 and $500 \mathrm{~nm}$ is attributed to the egg binder, and it is consistent with PL results and previously published studies [45-47]. In relative terms, 
and upon excitation at both 266 and $355 \mathrm{~nm}$, the fluorescence attributed to the egg binder in the spectrum of the yellow sample appears weaker than the emission at longer wavelengths, as compared with the blue and red ones. This is due to the contribution of the cadmium yellow pigment, centered at $520 \mathrm{~nm}$ (NBE) [49], which is enhanced by the excitation laser fluences used for LIF measurements.

Regarding the red sample, the spectra show a weak shoulder centered at around $585 \mathrm{~nm}$, superimposed on the tail of the binder fluorescence, especially visible upon excitation at $355 \mathrm{~nm}$ (Figure $4 \mathrm{~b}$ ). In this case, the contribution from the binder could be filtered and modified in shape by the red pigment absorption but, according to previous LIF studies of this pigment in its raw form or in mixtures with binders $[42,49,50]$, the feature at around $585 \mathrm{~nm}$ could be ascribed to the NBE emission of the semiconductor and/or irregularities in the crystal structure and presence of impurities, in agreement with the PL results. In turn, the LIF spectra of Egyptian blue includes the emission of the binder, although the contribution of additional pigment emission in this region cannot be ruled out [42].

The LIF emissions detected for the three colored samples fall into the detection range of the nonlinear optical microscopes used in this work, implying that the possible MPEF signals would result in contributions from both the binder and the pigments, although to different extents for each colored tempera.

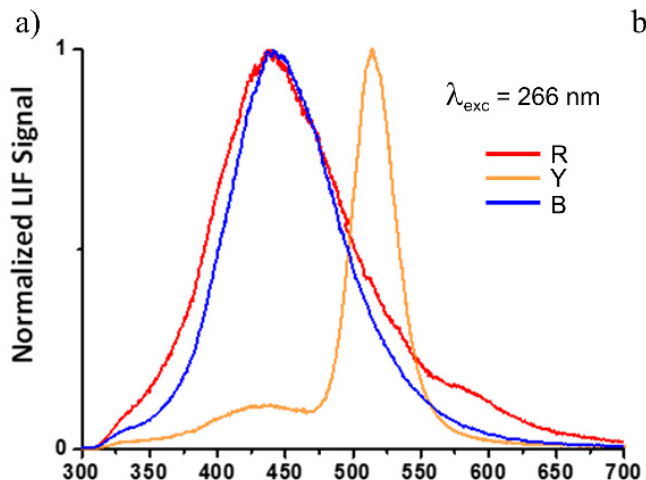

b)

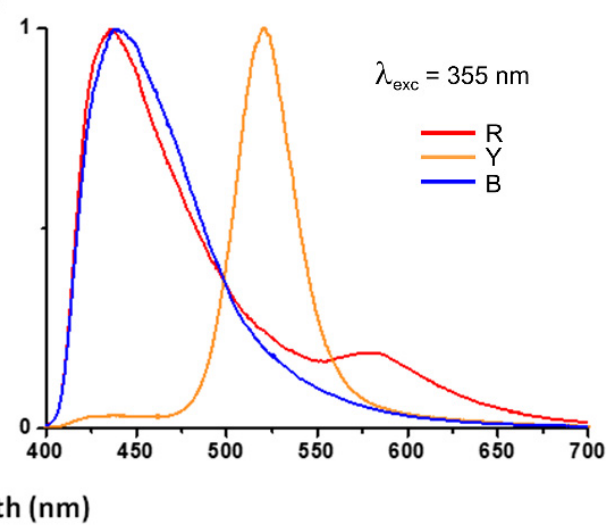

Figure 4. Laser Induced Fluorescence (LIF) spectra acquired by laser excitation at $266 \mathrm{~nm}(\mathbf{a})$ and $355 \mathrm{~nm}(\mathbf{b})$ of red, yellow, and blue tempera samples.

\subsection{Optical Coherence Tomography}

OCT results did not enable visualization of the interface between the paint and the preparation layers, and therefore did not allowing the measurement of the thickness of the former (Figure 5). This was attributed to the significant refractive index mismatch between pigment particles and egg binder, causing (especially in the case of the red and yellow pigments) an intense multiple scattering of the incident and reflected light components. In the case of Egyptian blue, the OCT probing light was able to penetrate, due to the strong absorption, only down to the first $50 \mu \mathrm{m}$ of the paint, but even if the scattering was less intense in this case, it was not possible to discern the bottom of the paint layer.
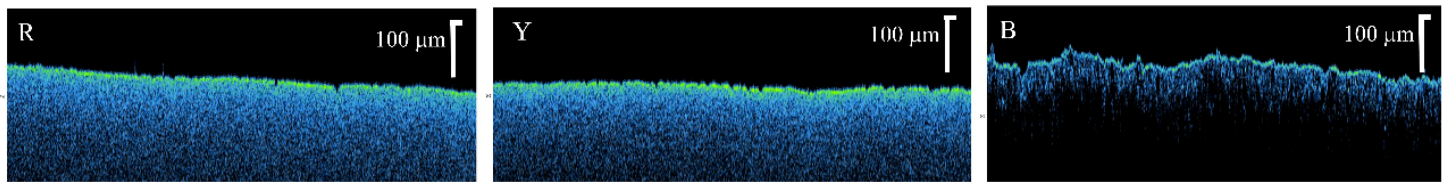

Figure 5. Optical Coherence Tomography (OCT) tomograms of red, yellow, and blue samples with vertical scale bars (in white). 
Given the above results and in order to determine the thickness of the paint layers, UV-Vis cross-section analysis was performed, in collaboration with the Opificio delle Pietre Dure (OPD) Scientific Laboratory (Florence, Italy), using a modular microscope (Carl Zeiss MicroImaging) equipped with a Colibri LED light source system and an externally coupled metal halide (HXP) white light source. Micro-samples were taken from the edge of each sample and subsequently embedded in polyester resin. According to the measurements performed (Figure 6), the thickness of the painted layers resulted in $48 \pm 3 \mu \mathrm{m}, 53 \pm 7 \mu \mathrm{m}$, and $98 \pm 7 \mu \mathrm{m}$ for red, yellow, and blue temperas, respectively.
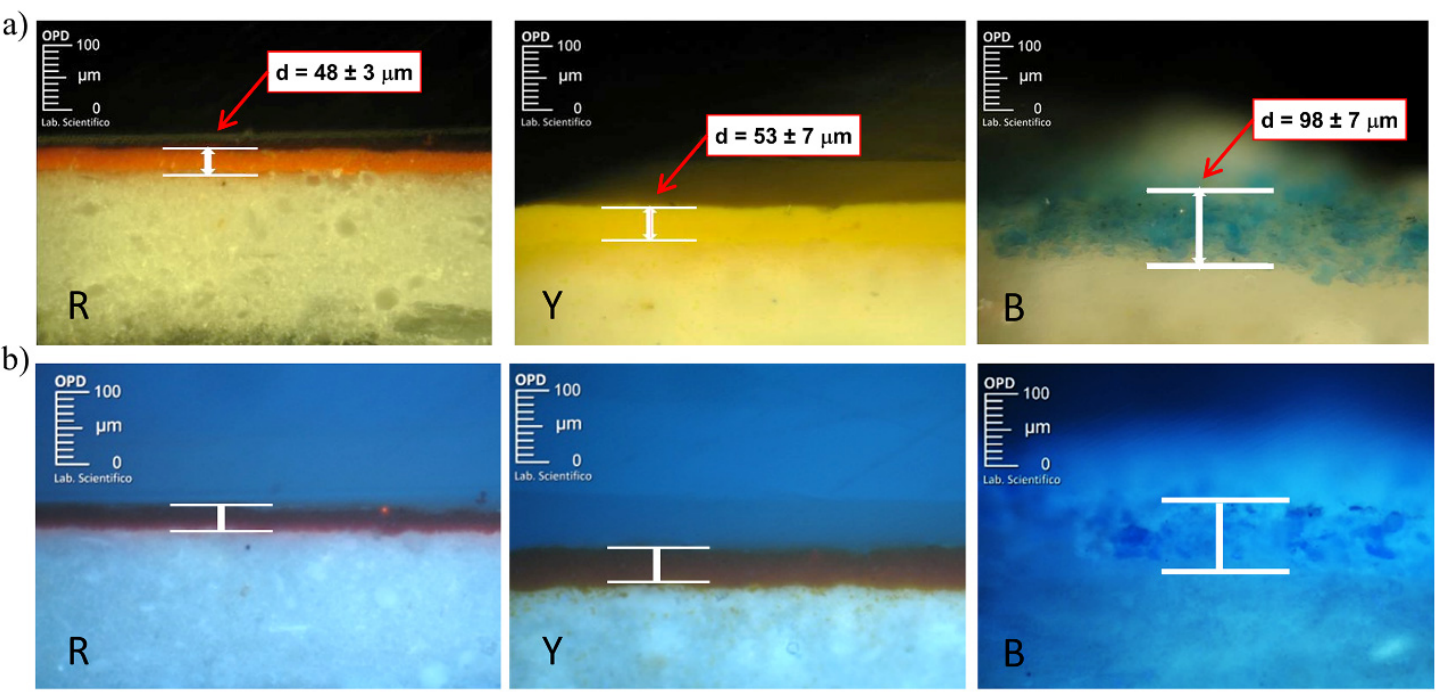

Figure 6. Cross-section images of R, Y, and B samples under visible (a) and UV (b) light (magnification $20 \times)$, showing the thickness of the paint layers.

\subsection{Multi-photon Excitation Fluorescence}

Based on the fluorescence behavior examined by PL and LIF (Sections 4.2 and 4.3), the excitation wavelengths chosen for MPEF analysis were expected to generate nonlinear signals in both the binder and the pigments, which were likely to fall in the detection range of the nonlinear optical microscopes used. Moreover, the reflectance and absorption properties analyzed in Section 4.1 suggested that a possible scattering of the incident laser light and/or an attenuation of the generated nonlinear signal should be taken into account during the analysis.

MPEF imaging performed with the IESL system upon excitation at $1028 \mathrm{~nm}$ did not allow for the measurement of the paint layers' thickness, and this was attributed to auto-absorption of the generated nonlinear signal from deeper layers and to the concurrent intense scattering of laser light obstructing its penetration. However, the emission generated at the surface of each sample enabled the collection of multi-photon fluorescence images and thus allowed visualization of the micro-features of each sample characterized by micrometric sized pigments' particles and their distribution. A consistent variability in signal intensity was observed, due to the different optical properties of each paint (Figure 7a). Specifically, the most intense emission was detected in the yellow sample, whereas weaker nonlinear signals were collected from the red and blue samples, in accordance with the fluorescent behavior analyzed in PL and LIF analysis. The structural morphology of the surface of the samples was screened by stereomicroscopy (representative stereomicrographs are reported in Figure $7 \mathrm{~b}$ ), showing the micrometric roughness of each paint surface and highlighting the presence of microgrooves on the red and yellow paints. 
a)

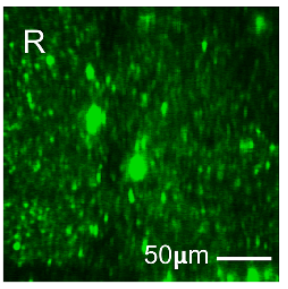

b)

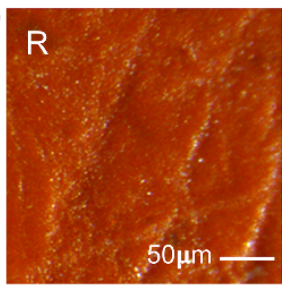

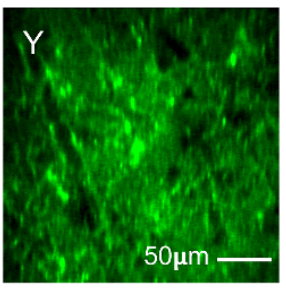
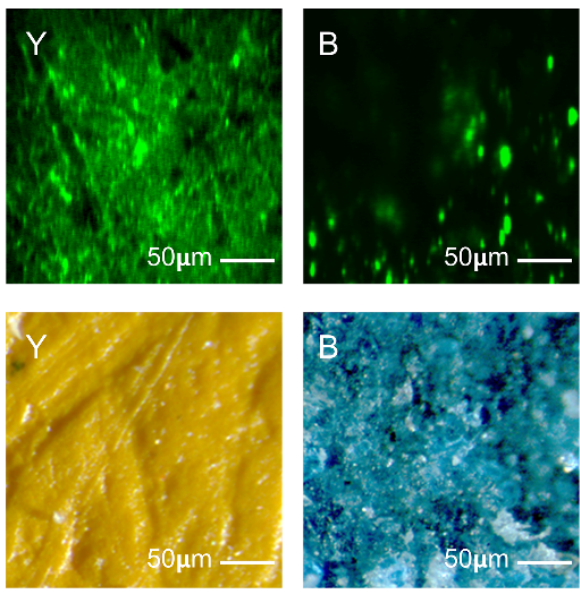

Figure 7. (a) MPEF imaging of the examined samples excited with a laser wavelength of $1028 \mathrm{~nm}$.

(b) Stereomicrographs of tempera samples.

The in-depth analysis of paint layers by MPEF was performed using the $800 \mathrm{~nm}$ excitation wavelength of the IQFR and INO setups. For a proper comparison with the previously applied techniques, measurements were carried out in the same selected area of the samples. As indicated in Section 3.5, and based on the signal intensity of each colored paint, detection was optimized by setting the laser power to the most suitable range, though it never exceeded $10 \mathrm{~mW}$ in order to ensure safe measuring conditions $[18,19]$. MPEF intensity profiles were measured as a function of depth, normalized, and fitted with a Lorentzian function. For all the profiles, the full width at half maximum (FWHM) was taken as an estimation of the apparent thickness of the paint layer. The real thickness was then calculated by correcting the FWHM values with the apparent depth correction factor (F) [27,51], which considers the refractive index of the analyzed material $(n=1.5-1.6)$ and the effective numerical aperture (NA) of the focusing objective lens ( 0.42 or 0.45 , depending on the setup). Thickness values provided by the IQFR nonlinear microscope resulted in agreement with cross-section analysis in the case of the blue sample $(116 \pm 5 \mu \mathrm{m})$, although values obtained for the red and yellow samples $(17 \pm 5 \mu \mathrm{m}$ and $14 \pm 5 \mu \mathrm{m}$, respectively) were underestimated. Thickness values measured with the INO setup showed good agreement with the cross-sections for all three colored samples. Representative MPEF imaging data acquired with the INO microscope are reported in Figure 8. MPEF emitted by each paint layer was measured in regions of interest $\left(\mathrm{ROI}=200 \times 200 \mu \mathrm{m}^{2}\right)$ with a scanning z-step of $1 \mu \mathrm{m}$. MPEF z-profiles extracted from the stack images are shown together with their respective fit functions (Figure 8a-c). The measured FWHM values, after refractive index correction, resulted in values of $45 \pm 5 \mu \mathrm{m}, 48 \pm 5 \mu \mathrm{m}$, and $103 \pm 5 \mu \mathrm{m}$, for red, yellow, and blue paints, respectively, which was in good agreement with the paint thickness measurements by UV-Vis cross-section analysis. Fluorescence intensity xy images (Figure $8 \mathrm{~d}-\mathrm{f}$ ), corresponding to the maximum signal intensity registered in the in-depth scanning, are reported together with the respective volume reconstructions (Figure $8 \mathrm{~g}-\mathrm{i}$ ) to allow the 3D visualization of both the morphology and the thickness of each paint layer. 

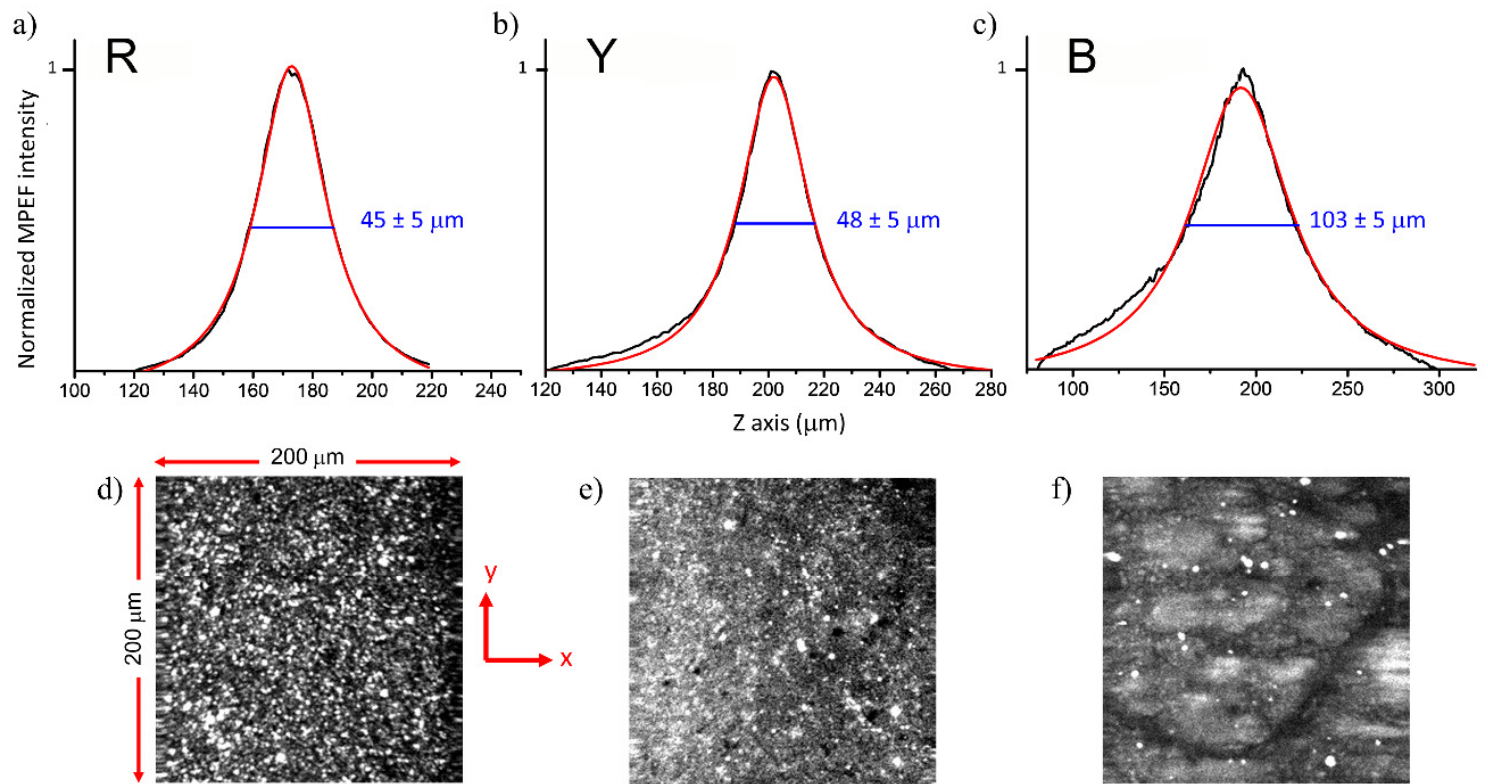

f)

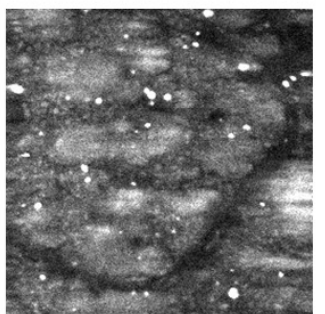

g)

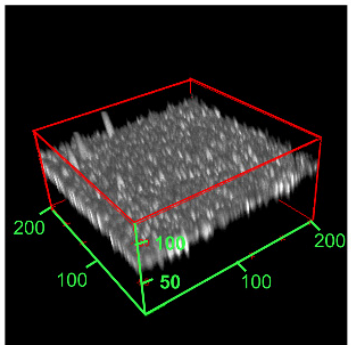

h)

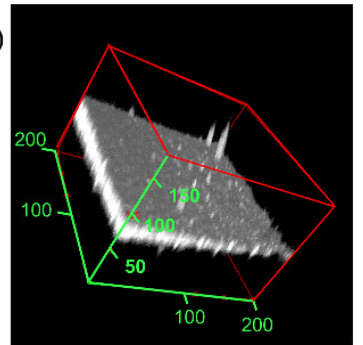

i)

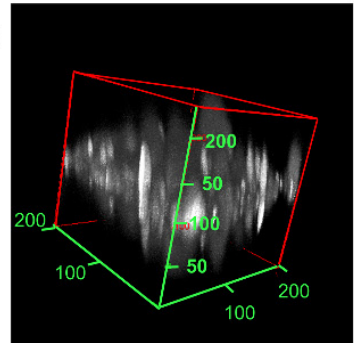

Figure 8. MPEF imaging results: (a-c) z-scans of the MPEF signals of $R, Y, B$ (in black), fits by Lorentzian functions (in red), and full width at half maximum (FWHM) values after refractive index correction, corresponding to the paint thickness (indicated in blue); (d-f) fluorescence intensity xy images $(200 \times 200 \mu \mathrm{m}, 256 \times 256$ pixels) extracted from the MPEF stacks at a depth corresponding to the maximum signal intensity; (g-i) Three-dimensional (3D) fluorescence reconstructions showing the thickness of each paint layer.

\section{Discussion and Conclusions}

In this study, we have evaluated the usefulness of MPEF microscopy for the analysis of egg-tempera paint systems simulating real paintings. Previous and complementary characterization of the pictorial materials was obtained using a multi-analytical approach, where the spectral information provided through FORS, LIF, and PL measurements allowed determination of the NLOM measurement conditions and supported the interpretation of the results.

Three different NLOM systems were tested; one with a laser excitation wavelength of $1028 \mathrm{~nm}$ and detection in the visible range, and the other two with excitation at $800 \mathrm{~nm}$ and detection in the 350-650 nm range. No MPEF profiles could be obtained with the former (IESL system), due to the strong scattering at the surface of the samples resulting from the $1028 \mathrm{~nm}$ excitation. However, the other two nonlinear microscopes based on $800 \mathrm{~nm}$ excitation provided MPEF profiles for all three colored samples. A possible explanation for this discrepancy is that two- or three-photon fluorescence excitation processes can be more effectively initiated by employing the shorter excitation wavelength $(800 \mathrm{~nm})$. Moreover, according to the spectral behavior of the three paints, the nonlinear optical signals generated after the interaction with this excitation wavelength likely undergo negligible auto-absorption while transmitting through the paint layer, thus allowing the signal detection. In respect of the two systems employing $800 \mathrm{~nm}$ excitation, the INO setup allowed measurement of the thickness of the paints, resulting in good agreement with the results obtained by UV-Vis cross-section analysis. The thickness 
values provided by the IQFR setup compared well with those retrieved by UV-VIS cross section for the blue sample but resulted in underestimation for the red and yellow paints. These diverging results may be explained by considering the scanning modality of the two setups (area-wise versus point-wise and for the INO and IQFR setups, respectively), which reflects in significantly different photon doses (200 pulses/point and $80 \times 10^{6}$ pulses/point for INO and IQFR, respectively). Although both nonlinear systems used the same laser power (which was the minimum necessary to obtain a measurable MPEF signal), the dissimilarly applied photon doses could have affected, to a diverse extent, the chemical stability of the paint layers according to the chemical composition of the pigments. Specifically, we hypothesize that the high photon dose applied with the IQFR setup could have caused chemical alterations in the red and yellow paints, thus providing underestimated measurements of thickness. Red lead is known to be naturally unstable under laser irradiation, causing a blackening effect mainly due to the transformation into plattnerite $\left(\mathrm{PbO}_{2}\right)$ or galena ( $\left.\mathrm{PbS}\right)$ [52]. Similarly, the low thickness value obtained for the yellow tempera under irradiation with high photon dose is likely due to photo-oxidative degradation of the pigment, the semiconductor cadmium sulphide [53]. A deeper investigation on these possible laser-induced degradation effects is envisaged for future studies. Conversely, we assume that no photochemical alteration was produced in the Egyptian blue tempera, given the well-known high photostability of this pigment [54]. In fact, the thickness of the blue tempera paint of around 100 microns could be measured with both NLOM systems.

Based on these results, we can conclude that, for tempera paints such as the ones studied here, cross-sectional analysis by MPEF yields satisfactory results if the photon dose is set below safe limits. This parameter is as crucial as the laser power applied, as already stated in [19]. Moreover, photochemical changes inside the focal volume of the laser beam should be monitored during MPEF measurements by simply observing the variation in the signal intensity, which has been proven to be a sign of possible damage [19]. This can be achieved by using an imaging NLOM system enabling faster scanning and quasi-real-time visual monitoring.

With this contribution, we have demonstrated the capability of MPEF in overcoming the detection limit of other linear optical techniques in probing highly scattering media, while ensuring the minimal photochemical disturbance to the examined materials. To the best of our knowledge, this is the first time that the potential of MPEF to provide 3D surveys of mock-up paintings has been extensively explored through the synergic application of a number of non-invasive techniques and through the comparison of three different nonlinear optical microscope setups.

Author Contributions: M.S. (Mikel Sanz) and M.O. performed the investigation and contributed to the writing; R.F., P.T., R.C., A.R., and G.F. supervised the investigation and reviewed the manuscript; M.S. (Marcin Sylwestrzak), S.M., C.G., S.P., and A.L. performed the investigation; M.C. administrated the project, supervised the investigation, contributed to the writing and reviewed the manuscript; A.D.F. performed the investigation, assembled the results, wrote and edited the manuscript. All authors have read and agreed to the published version of the manuscript.

Funding: This research was funded by the European H2020 IPERION CH Project (Integrated Platform for the European Research Infrastructure ON Cultural Heritage (GA 654028, WP6); Spanish State Research Agency (AEI) and European Regional Development (FEDER) through Project CTQ2016-75880-P-AEI/FEDER, UE; and TOP Heritage-CM (S2018/NMT-4372) from the Community of Madrid and with the support of Plataforma Temática Interdisciplinar of CSIC "Patrimonio Abierto: Investigación y Sociedad" (PTI-PAIS).

Acknowledgments: Useful discussions with D. Anglos (IESL-FORTH, Greece) are gratefully acknowledged.

Conflicts of Interest: The authors declare no conflict of interest.

\section{References}

1. Zipfel, W.R.; Williams, R.M.; Webb, W.W. Nonlinear magic: Multiphoton microscopy in the biosciences. Nat. Biotechnol. 2003, 21, 1369-1377. [CrossRef]

2. Filippidis, G.; Tserevelakis, G.; Selimis, A.; Fotakis, C. Nonlinear imaging techniques as non-destructive, high-resolution diagnostic tools for cultural heritage studies. Appl. Phys. A 2014, 118, 417-423. [CrossRef]

3. Latour, G.; Echard, J.-P.; Didier, M.; Schanne-Klein, M.-C. In situ 3D characterization of historical coatings and wood using multimodal nonlinear optical microscopy. Opt. Express 2012, 20, 24623. [CrossRef] 
4. Psilodimitrakopoulos, S.; Gavgiotaki, E.; Melessanaki, K.; Tsafas, V.; Filippidis, G. Polarization Second Harmonic Generation Discriminates Between Fresh and Aged Starch-Based Adhesives Used in Cultural Heritage. Microsc. Microanal. 2016, 22, 1072-1083. [CrossRef]

5. Filippidis, G.; Massaouti, M.; Selimis, A.; Gualda, E.J.; Manceau, J.-M.; Tzortzakis, S. Nonlinear imaging and THz diagnostic tools in the service of Cultural Heritage. Appl. Phys. A 2011, 106, 257-263. [CrossRef]

6. Gueta, R.; Tal, E.; Silberberg, Y.; Rousso, I. The 3D structure of the tectorial membrane determined by second-harmonic imaging microscopy. J. Struct. Boil. 2007, 159, 103-110. [CrossRef]

7. Dombeck, D.A.; Blanchard-Desce, M.; Webb, W.W. Optical Recording of Action Potentials with Second-Harmonic Generation Microscopy. J. Neurosci. 2004, 24, 999-1003. [CrossRef] [PubMed]

8. Denk, W.; Strickler, J.; Webb, W. Two-photon laser scanning fluorescence microscopy. Science 1990, 248, 73-76. [CrossRef]

9. Diaspro, A.; Bianchini, P.; Vicidomini, G.; Faretta, M.; Ramoino, P.; Usai, C. Multi-photon excitation microscopy. Biomed. Eng. Online 2006, 5, 36. [CrossRef] [PubMed]

10. Filippidis, G.; Gualda, E.J.; Melessanaki, K.; Fotakis, C. Nonlinear imaging microscopy techniques as diagnostic tools for art conservation studies. Opt. Lett. 2008, 33, 240-242. [CrossRef] [PubMed]

11. Mari, M.; Tsafas, V.; Melessanaki, K.; Filippidis, G. Applications of non-linear imaging microscopy techniques to cultural heritage objects. Insight - Non-Destr. Test. Cond. Monit. 2018, 60, 663-669. [CrossRef]

12. Targowski, P.; Iwanicka, M. Optical Coherence Tomography: Its role in the non-invasive structural examination and conservation of cultural heritage objects-A review. Appl. Phys. A 2011, 106, 265-277. [CrossRef]

13. Targowski, P.; Iwanicka, M.; Rouba, B.J.; Frosinini, C. OCT for Examination of Artwork. In Biomaterials; Springer Science and Business Media LLC: Berlin, Germany, 2015; pp. 2473-2495.

14. Fontana, R.; Dal Fovo, A.; Striova, J.; Pezzati, L.; Pampaloni, E.; Raffaelli, M.; Barucci, M. Application of non-invasive optical monitoring methodologies to follow and record painting cleaning processes. Appl. Phys. A 2015, 121, 957-966. [CrossRef]

15. Liang, H.; Cid, M.G.; Cucu, R.G.; Dobre, G.M.; Podoleanu, A.; Pedro, J.; Saunders, D. En-face optical coherence tomography-A novel application of non-invasive imaging to art conservation. Opt. Express 2005, 13, 6133. [CrossRef] [PubMed]

16. Striova, J.; Dal Fovo, A.; Fontani, V.; Barucci, M.; Pampaloni, E.; Raffaelli, M.; Fontana, R. Modern acrylic paints probed by optical coherence tomography and infrared reflectography. Microchem. J. 2018, 138, 65-71. [CrossRef]

17. Iwanicka, M.; Sylwestrzak, M.; Targowski, P. Optical Coherence Tomography (OCT) for Examination of Artworks. In Advanced Characterization Techniques, Diagnostic Tools and Evaluation Methods in Heritage Science; Springer Science and Business Media LLC: Berlin, Germany, 2018; pp. 49-59.

18. Liang, H.; Mari, M.; Cheung, C.S.; Kogou, S.; Johnson, P.; Filippidis, G. Optical coherence tomography and non-linear microscopy for paintings-A study of the complementary capabilities and laser degradation effects. Opt. Express 2017, 25, 19640. [CrossRef]

19. Dal Fovo, A.; Sanz, M.; Mattana, S.; Oujja, M.; Marchetti, M.; Pavone, F.; Cicchi, R.; Fontana, R.; Castillejo, M. Safe limits for the application of nonlinear optical microscopies to cultural heritage: A new method for in-situ assessment. Microchem. J. 2020, 154, 104568. [CrossRef]

20. Mari, M.; Filippidis, G. Non-Linear Microscopy: A Well-Established Technique for Biological Applications towards Serving as a Diagnostic Tool for in situ Cultural Heritage Studies. Sustainability 2020, 12, 1409. [CrossRef]

21. Selimis, A.; Vounisiou, P.; Tserevelakis, G.; Melessanaki, K.; Pouli, P.; Filippidis, G.; Beltsios, C.; Georgiou, S.; Fotakis, C. In-depth assessment of modifications induced during the laser cleaning of modern paintings. SPIE Eur. Opt. Metrol. 2009, 7391, 73910. [CrossRef]

22. Vounisiou, P.; Selimis, A.; Tserevelakis, G.; Melessanaki, K.; Pouli, P.; Filippidis, G.; Beltsios, C.; Georgiou, S.; Fotakis, $\mathrm{C}$. The use of model probes for assessing in depth modifications induced during laser cleaning of modern paintings. Appl. Phys. A 2010, 100, 647-652. [CrossRef]

23. Filippidis, G.; Mari, M.; Kelegkouri, L.; Philippidis, A.; Selimis, A.; Melessanaki, K.; Sygletou, M.; Fotakis, C. Assessment of In-Depth Degradation of Artificially Aged Triterpenoid Paint Varnishes Using Nonlinear Microscopy Techniques. Microsc. Microanal. 2014, 21, 510-517. [CrossRef] 
24. Oujja, M.; Psilodimitrakopoulos, S.; Carrasco, E.; Sanz, M.; Philippidis, A.; Selimis, A.; Pouli, P.; Filippidis, G.; Castillejo, M. Nonlinear imaging microscopy for assessing structural and photochemical modifications upon laser removal of dammar varnish on photosensitive substrates. Phys. Chem. Chem. Phys. 2017, 19, 22836-22843. [CrossRef]

25. Faraldi, F.; Tserevelakis, G.; Filippidis, G.; Ingo, G.M.; Riccucci, C.; Fotakis, C. Multi photon excitation fluorescence imaging microscopy for the precise characterization of corrosion layers in silver-based artifacts. Appl. Phys. A 2013, 111, 177-181. [CrossRef]

26. Villafana, T.E.; Brown, W.P.; Delaney, J.K.; Palmer, M.; Warren, W.S.; Fischer, M.C. Femtosecond pump-probe microscopy generates virtual cross-sections in historic artwork. Proc. Natl. Acad. Sci. USA 2014, 111, 1708-1713. [CrossRef]

27. Dal Fovo, A.; Oujja, M.; Sanz, M.; Martínez-Hernández, A.; Cañamares, M.V.; Castillejo, M.; Fontana, R. Multianalytical non-invasive characterization of phthalocyanine acrylic paints through spectroscopic and non-linear optical techniques. Spectrochim. Acta Part A: Mol. Biomol. Spectrosc. 2019, 208, 262-270. [CrossRef]

28. Dal Fovo, A.; Striova, J.; Pampaloni, E.; Barucci, M.; Raffaelli, M.; Mercatelli, R.; Pezzati, L.; Cicchi, R. Nonlinear optical imaging techniques (NLO) for painting investigation. Lasers Conserv. Artworks XI 2017. [CrossRef]

29. Dupuis, G.; Menu, M. Quantitative characterisation of pigment mixtures used in art by fibre-optics diffuse-reflectance spectroscopy. Appl. Phys. A. 2006, 83, 469-474. [CrossRef]

30. Romani, A.; Clementi, C.; Miliani, C.; Favaro, G. Fluorescence Spectroscopy: A Powerful Technique for the Noninvasive Characterization of Artwork. Accounts Chem. Res. 2010, 43, 837-846. [CrossRef] [PubMed]

31. Anglos, D.; Solomidou, M.; Zergioti, I.; Zafiropulos, V.; Papazoglou, T.; Fotakis, C. Laser-Induced Fluorescence in Artwork Diagnostics: An Application in Pigment Analysis. Appl. Spectrosc. 1996, 50, 1331-1334. [CrossRef]

32. Targowski, P.; Kowalska, M.; Sylwestrzak, M.; Iwanicka, M. OCT for Examination of Cultural Heritage Objects; Wang, M., Ed.; IntechOpen: London, UK; Rijeka, Croatia, 2020; in press. [CrossRef]

33. Psilodimitrakopoulos, S.; Mouchliadis, L.; Paradisanos, I.; Lemonis, A.; Kioseoglou, G.; Stratakis, E. Ultrahigh-resolution non-linear optical imaging of the armchair orientation in $2 \mathrm{D}$ transition metal dichalcogenides. Light-Sci Appl. 2018, 7, 18005. [CrossRef]

34. Psilodimitrakopoulos, S.; Mouchliadis, L.; Paradisanos, I.; Kourmoulakis, G.; Lemonis, A.; Kioseoglou, G.; Stratakis, E. Twist Angle mapping in layered WS2 by Polarization-Resolved Second Harmonic Generation. Sci. Rep. 2019, 9, 14285. [CrossRef] [PubMed]

35. Marchetti, M.; Baria, E.; Cicchi, R.; Pavone, F. Custom Multiphoton/Raman Microscopy Setup for Imaging and Characterization of Biological Samples. Methods Protoc. 2019, 2, 51. [CrossRef] [PubMed]

36. Mercatelli, R.; Mattana, S.; Capozzoli, L.; Ratto, F.; Rossi, F.; Pini, R.; Fioretto, D.; Pavone, F.S.; Caponi, S.; Cicchi, R. Morpho-mechanics of human collagen superstructures revealed by all-optical correlative micro-spectroscopies. Commun. Boil. 2019, 2, 117. [CrossRef] [PubMed]

37. Zhou, Y.; Long, J.; Gu, Q.; Lin, H.; Lin, H.; Wang, X. Photoinduced Reactions between Pb3O4 and Organic Dyes in Aqueous Solution under Visible Light. Inorg. Chem. 2012, 51, 12594-12596. [CrossRef] [PubMed]

38. Rosi, F.; Grazia, C.; Gabrieli, F.; Romani, A.; Paolantoni, M.; Vivani, R.; Brunetti, B.G.; Colomban, P.; Miliani, C. UV-Vis-NIR and micro Raman spectroscopies for the non destructive identification of Cd 1-x Zn x S solid solutions in cadmium yellow pigments. Microchem. J. 2016, 124, 856-867. [CrossRef]

39. Grazia, C.; Rosi, F.; Gabrieli, F.; Romani, A.; Paolantoni, M.; Vivani, R.; Brunetti, B.G.; Colomban, P.; Miliani, C. UV-Vis-NIR and microRaman spectroscopies for investigating the composition of ternary CdS $1-\mathrm{x}$ Se $\mathrm{x}$ solid solutions employed as artists' pigments. Microchem. J. 2016, 125, 279-289. [CrossRef]

40. Accorsi, G.; Verri, G.; Bolognesi, M.; Armaroli, N.; Clementi, C.; Miliani, C.; Romani, A. The exceptional near-infrared luminescence properties of cuprorivaite (Egyptian blue). Chem. Commun. 2009, 23, 3392. [CrossRef]

41. Pozza, G.; Ajo, D.; Chiari, G.; De Zuane, F.; Favaro, M. Photoluminescence of the inorganic pigments Egyptian blue, Han blue and Han purple. J. Cult. Herit. 2000, 1, 393-398. [CrossRef]

42. Mounier, A.; Lazare, S.; Le Bourdon, G.; Aupetit, C.; Servant, L.; Daniel, F. LED $\mu$ SF: A new portable device for fragile artworks analyses. Application on medieval pigments. Microchem. J. 2016, 126, 480-487. [CrossRef]

43. De La Rie, E.R. Fluorescence of paint and varnish layers (Part 1). Stud. Conserv. 1982, 27, 1-7. [CrossRef] 
44. Clementi, C.; Rosi, F.; Romani, A.; Vivani, R.; Brunetti, B.G.; Miliani, C. Photoluminescence Properties of Zinc Oxide in Paints: A Study of the Effect of Self-Absorption and Passivation. Appl. Spectrosc. 2012, 66, 1233-1241. [CrossRef]

45. Nevin, A.; Anglos, D. Assisted Interpretation of Laser-Induced Fluorescence Spectra of Egg-Based Binding Media Using Total Emission Fluorescence Spectroscopy. Laser Chem. 2006, 2006, 1-5. [CrossRef]

46. Nevin, A.; Cather, S.; Anglos, D.; Fotakis, C. Analysis of protein-based binding media found in paintings using laser induced fluorescence spectroscopy. Anal. Chim. Acta 2006, 573, 341-346. [CrossRef]

47. Pelagotti, A.; Pezzati, L.; Bevilacqua, N.; Vascotto, V.; Reillon, V.; Daffara, C. A study of UV fluorescence emission of painting materials. In Proceedings of the Art'05-8th International Conference on Non Destructive Investigations and Microanalysis for the Diagnostics and Conservation of the Cultural and Environmental Heritage, Lecce, Italy, 15-19 May 2005.

48. Thoury, M.; Delaney, J.K.; De La Rie, E.R.; Palmer, M.; Morales, K.; Krueger, J. Near-Infrared Luminescence of Cadmium Pigments: In Situ Identification and Mapping in Paintings. Appl. Spectrosc. 2011, 65, 939-951. [CrossRef]

49. Borgia, I.; Fantoni, R.; Flamini, C.; Di Palma, T.M.; Guidoni, A.G.; Mele, A. Luminescence from pigments and resins for oil paintings induced by laser excitation. Appl. Surf. Sci. 1998, 127, 95-100. [CrossRef]

50. Lopez, M.; Bai, X.; Koch-Dandolo, C.; Serfaty, S.; Wilkie-Chancellier, N.; Detalle, V. Nd:YAG vs Er:YAG: A comparative study of laser varnish removal on easel paintings. Opt. Arts Archit. Archaeol. VII 2019, 1105804. [CrossRef]

51. White, G.W. Improving the accuracy of vertical measurements under the microscope. Microscope 1970, 18, 51-59.

52. Pouli, P.; Emmony, D. The effect of Nd:YAG laser radiation on medieval pigments. J. Cult. Herit. 2000, 1, S181-S188. [CrossRef]

53. Comelli, D.; MacLennan, D.; Ghirardello, M.; Phenix, A.; Patterson, C.S.; Khanjian, H.; Gross, M.; Valentini, G.; Trentelman, K.; Nevin, A. Degradation of Cadmium Yellow Paint: New Evidence from Photoluminescence Studies of Trap States in Picasso's Femme (Époque des “Demoiselles d'Avignon"). Anal. Chem. 2019, 91, 3421-3428. [CrossRef]

54. Berke, H. The invention of blue and purple pigments in ancient times. Chem. Soc. Rev. 2007, 36, 15-30. [CrossRef] 